\title{
Über Adenase und ihre Beziehung zu der Entstehung von Hypoxanthin im Organismus. \\ Von
}

Carl Vögtlin und Walter Jones.

(Aus den Laboratorien für Pharmakologie und physiologische Chemie, Johns Hopkins University).

(Der Redaktion zugègangen am 28. März 1910.)

Im Verlaufe seiner bekannten Arbeit über den endogenen Ursprung der Harnsäure bemerkt Burian, ${ }^{1}$ ) daß, wenn man das Hypoxanthin aus einem überlebenden Muskel eines Hundes durch Perfusion entfernt, an Stelle davon eine gleiche Menge dieser Base in dem Muskel produziert wird, sodaß die Menge dieses Bestandteils in dem Gewebe beständig zu bleiben strebt. Seine Experimente waren selbstverständlich so gehalten, daß eine Einwanderung von Hypoxanthin aus anderen Teilen des Körpers ausgeschlossen war. Daraus schließt er, daß die Base im lebenden Körper fortwährend gebildet wird aus einem unbekannten Vorläufer, der kein Bestandteil der Leukocyten ist. Da die Menge des Hypoxanthins in der Perfusionsflüssigkeit sehr viel größer war nach Reiz, als nach einer Ruheperiode, nimmt Buria n einen sicheren kausalen Zusammenhang zwischen Muskelreiz und der Produktion von Muskel-Hypoxanthin an.

Kurz vor dem Erscheinen von Burians Publikation wurde gezeigt, daß das Hypoxanthin aus Adenin gebilket werden kann durch die desamidierende Wirkung eines Ferments, ${ }^{2}$ ) Adenase, welches in den wässerigen Lösungen einer Anzahl tierischer Organe aktiv ist. Die weitere Untersuchung von diesem und einem andern weit verbreiteten Ferment, der Guanase, ${ }^{8}$ ) hat Resultate gegeben, welche die Bildung von Harnsäure aus Leukocyten so gut erklären konnten, daß die Aufmerksamkeit

1) Burian, Diese Zeitschrift, Bd. XLIII, S. 532.

2) Jones, Diese Zeitschrift, Bd. XLI, S. 101; Bd. XLII,.S. 35. Jones und Winternitz, Bd. XLIV, S. 1.

$\left.{ }^{3}\right)$ Jones und Partridge, Diese Zeitschrift, Bd. XLII, S. 343. 
einige Zeit von Burians Arbeit abgelenkt wurde und man die Anwesenheit von Hypoxanthin in einem Organextrakt mehr oder weniger als Beweis für die Existenz von Adenase im Gewebe angesehen hat. In der folgenden kurzen Mitteilung beabsichtigen wir, einen starken Beweis zu liefern, daß die Entstehung von Hypoxanthin im Muskel unzweifelhaft bei Abwesenheit von Adenase vor sich geht.

Es wird anerkannt, daß ein wässeriges Extrakt von Hundemuskel entweder frei ist von Adenase, ${ }^{1}$ ) oder daß deren Wirkung so gering ist, daß auf diese schwache Wirkung speziell Nachdruck gelegt werden muß. ${ }^{2}$ ) Natürlich kann die Behauptung, daß ein Ferment aus einem bestimmten Gewebe abwesend ist, nur bedeuten, daß die analytischen Methoden, über die wir verfügen, nicht genau genug sind, um in einer unverhältnismäßig langen Zeit die Veränderungen, die das Ferment verursacht; zu entdecken. Auch schließt die Annahme, daß ein Ferment abwesend sei, nicht aus, daß so wenig davon da ist, daß Bedingungen eintreten, die seine Tätigkeit verhindern, ehe es Zeit gehabt hat, sich bemerkbar zu machen. Aus Überlegungen dieser Art schien es rätlich, die Wirkung der Adenase in einem fermentarmen Gewebsextrakt zu beobachten, um zu sehen, ob irgendsolche retardierende oder zerstörende Faktoren entstehen. Es ist nicht leicht, einen Gewebsextrakt zu finden, der zu diesem Zweck gut geeignet ist. Im Vergleich mit der weit verbreiteten Guanase kommt eine aktive Form der Adenase selten vor. Man findet sie in den Organen des Ochsen, aber das Ferment fehlt entweder ganz oder ist nur in geringen Spuren vorhanden in Extrakten der meisten Organe des Kaninchens, Hundes oder Menschen. Auch in der Hefe kann sein Vorkommen nicht deutlich bewiesen werden. Im Muskelgewebe, wo sich das Hypoxanthin hauptsächlich bildet; fehlt das Ferment ganz besonders. In Muskelextrakten vom Hund, Schwein, Menschen, Ratte, Kaninchen und Ochsen ist es uns nur bei der letzten Spezies gelungen, die Gegenwart der Adenase nachzuweisen. Bei manchen Tierarten kann das

1) Leonard und Jones, Journal of Biol. Chem., Bd. VI, S. 453.

2) Schittenhelm, Diese Zeitschrift, Bd. LXIII, S. 254. 
Ferment kaum in irgend einem Organextrakt nachgewiesen werden. Dies hat sich besonders bei der Ratte ${ }^{1}$ ) herausgestellt und stimmt gut zu Nicolaiers ${ }^{2}$ ) Beobachtung, daß bei Ratten subkutan injiziertes Adenin oxydiert wird, aber ohne Desamidierung $z u$ den Nieren gelangt, wo es einen Niederschlag von 6 Amido-2,8-dioxypurin veranlaßt, ${ }^{3}$ )

Nachdem wir eine größere Anzahl Gewebsextrakte untersucht hatten, fanden wir, daß Schweinemilz wohl geeignet für das Studium einer unzweifelhaften Spur von Adenase ist. Eine ungefähre Idee von der Armut dieses Gewebes an Adenasè kann gewonnen werden, wenn man seine Wirkung mit der der Guanase von Kaninchenleber vergleicht. $200 \mathrm{ccm}$ eines wässerigen Extraktes von Kaninchenleber mit 4 Teilen Wasser kann $100 \mathrm{mg}$ Guanin ganz in Xanthin verwandeln in weniger als 15 Stunden, bei Zimmertemperatur. (Wir wissen noch nicht, wie viel weniger als 15 Stunden.) Ein ähnlicher Extrakt von Schweinemilz bedarf ungefähr $6 \mathrm{mal}$ soviel Zeit, um dieselbe Menge Adenin bei Körpertemperatur zu verwandeln. Da wir gefunden haben, daß Adenase bei $40^{\circ}$ mindestens $30 \mathrm{mal}$ so aktiv ist, als bei $30^{\circ}$, so müßte man annehmen, daß die Reaktionsgeschwindigkeit in dem einen Fall $180 \mathrm{mal}$ so groß ist als in anderen. Jedoch ist die Gegenwart von Adenase in Schweinemilz unzweifelhaft. Ihre Wirkung ist bei $40^{\circ}$ schnell genug $\mathrm{zu}$ leichter Beobachtung und langsam genug, um nur als Spur des Fermentes betrachtet zu werden, wobei man erwartet, daß jeder Faktor, der eine merkliche Störung auf das Ferment ausübt, der geringen Aktivität des Gewebes ein Ende macht.

Ein wässeriges Extrakt von Schweinemilz wurde bereitet mit 4 Teilen Wasser, und nachdem es bei $20^{\circ} 8$ Stunden bei öfterem Schütteln gestanden hatte, wurde die wolkige Flüssigkeit abgeseiht (Chloroform wurde als Antiseptikum benützt). $\mathrm{Zu} 800 \mathrm{ccm}$ dieser Flüssigkeit wurde hinzugefügt $600 \mathrm{mg}$ Adeninsulfat, das

1) Rohde und Jones, Journ. of Biol. Chem., Bd. VII, S. 237, 1910.

2) Nicolaier, Zeitschrift f. klin. Med., Bd. XLV, S. 359.

s) Über neuere Literatur über die Verteilung der Purinfermenté siehe Wells, The Purine Metabolism of the Monkey, Journal of Biological Chemistry, Bd. VII, S. 171. 
in $100 \mathrm{ccm}$ Wasser bei $40^{\circ}$ gelöst war mit soviel Natronlauge, daß die Flüssigkeit neutral war. Das Gemisch wurde bei $40^{\circ}$ im Thermostaten digeriert, und nach verschiedenen Zeiträumen wurde ein Viertel des gut gemischten Produkts zur Untersuchung entnommen. Die Analyse wurde auf die gewöhnliche Art gemacht. Die Basen wurden erst als Kupferverbindungen, dann als Silberverbindungen niedergeschlagen und das Adenin wurde mit Pikrinsäure von dem Hypoxanthin getrennt. In dem Filtrat vom Adeninpikrat wurde der Purinstickstoff bestimmt und die entsprechende Menge Hypoxanthin berechnet. Die Resultate sind in folgender Tabelle gegeben:

\begin{tabular}{c|c|c|c|c|c}
\hline $\begin{array}{c}\text { Ursprünglich } \\
\text { vorhandenes } \\
\text { Adeninsulfat }\end{array}$ & $\begin{array}{c}\text { Daraus } \\
\text { berechnet } \\
\text { Adenin } \\
\mathrm{mg}\end{array}$ & $\begin{array}{c}\text { Zeit } \\
\text { der } \\
\text { Digestion } \\
\text { Stunden }\end{array}$ & $\begin{array}{c}\text { Adenin- } \\
\text { pikrat } \\
\mathrm{mg}\end{array}$ & $\begin{array}{c}\text { Daraus } \\
\text { berechnet } \\
\text { Adenin } \\
\mathrm{mg}\end{array}$ & $\begin{array}{c}\text { Hypo- } \\
\text { xanthin } \\
\mathrm{mg}\end{array}$ \\
\hline 150 & 100 & 19 & 203 & 75 & 18 \\
150 & 100 & 43 & 136 & 50 & 32 \\
$\therefore 150$ & 100 & 67 & 55 & 20 & 59 \\
150 & 100 & 91 & 0 & 0 & 91
\end{tabular}

Wenn man eine durchgeseihte Emulsion von Schweinemilz und Wasser mehrere Tage bei Zimmertemperatur stehen läßt, so scheidet sich aus der tief blutroten filtrierbaren Lösung ein rötlicher Niederschlag ab. Bei weiterem Stehen setzt sich die Bildung des Niederschlages von Eiweiß und Farbstoff fort, bis schließlich ein filtrierter Teil keine rote Farbe mehr hat, sondern nur blaßbraun ist. Dann hört der Niederschlag von Eiweiß auf und die Lösung kann wochenlang ohne anscheinende Veränderung aufgehoben werden. Man würde nicht erstaunt sein, zu finden, daß diese Veränderungen und das lange Stehen die Adenase zerstört hätten, aber die Resultate, die in der Tabelle weiter unten gegeben sind, zeigen, daß die Aktivität des Ferments nicht merklich verändert war. Die Lösung wurde hergestellt durch Filtration des trüben Extrakts, nachdem er 35 Tage bei $30^{\circ}$ gestanden hatte. Die Experimente wurden wie oben beschrieben angestellt und die Resultate waren genauer, 
weil bei Abwesenheit der von dem Extrakt abfiltrierten Eiweißstoffe die in das Gerinnsel eingeschlossenen Mengen nicht so groß waren.

\begin{tabular}{c|c|c|c|c|c}
\hline $\begin{array}{c}\text { Ursprünglich } \\
\text { vorhandenes } \\
\text { Adeninsulfat } \\
\text { mg }\end{array}$ & $\begin{array}{c}\text { Daraus } \\
\text { berechnet } \\
\text { Adenin } \\
\text { mg }\end{array}$ & $\begin{array}{c}\text { Zeit } \\
\text { der } \\
\text { Digestion } \\
\text { Stunden }\end{array}$ & $\begin{array}{c}\text { Adenin- } \\
\text { pikrat }\end{array}$ & $\begin{array}{c}\text { Daraus } \\
\text { berechnet } \\
\text { Adenin } \\
\text { mg }\end{array}$ & $\begin{array}{c}\text { Hypo- } \\
\text { xanthin } \\
\text { mg }\end{array}$ \\
\hline 150 & 100 & 24 & 218 & 81 & 21 \\
150 & 100 & 48 & 141 & 52 & 43 \\
150 & 100 & 72 & 62 & 21 & 71 \\
150 & 100 & 96 & 0 & 0 & 93
\end{tabular}

Während wir so die ununterbrochene Wirkung dieses spärlichen Auftretens von Adenase in Schweinemilz geuau beobachten konnten, war es Leonard und Jones unmöglich, irgend welche Tätigkeit eines solches Ferments in einem Extrakt von Hundemuskel zu entdecken, selbst nach einer Digestion von 25 Tagen. Es kann deshalb mit großer Wahrscheinlichkeit ausgesagt werden, daß das Hypoxanthin von Hundemuskel, dessen Zusammenhang mit dem spezifischen physiologischen Charakter des Gewebes Burian nachwies, keinen direkten Konnex mit dem Ferment Adenase besitzt. Man kann noch weiter gehen, denn nach einer physiologischen Betrachtung über Muskeln im allgemeinen kann man mit einer gewissen Berechtigung annehmen, daß die Schlußfolgerungen in bezug auf Hundemuskel auf alle Muskeln anzuwenden sind, sowohl die willkürlichen als die unwillkürlichen, sogar auf Ochsenmuskel, welcher Adenase enthält.

Um jeden Irrtum auszuschließen in einer Sache, die von so weiter Bedeutung sein kann, beschlossen wir zu untersuchen, ob überlebender Hundemuskel eine erkennbare Spur von Adenase enthält. $\mathrm{Zu}$ diesem Zweck wurden 3 Lösungen von defibriniertem Hundeblut hergestellt.

1. $200 \mathrm{ccm}$ Blut und $800 \mathrm{ccm}$ Ring ersche Lösung. Dies wurde verwendet, um das Gewebe sowohl vor als nach der. Perfusion auszuwaschen. 
2. $200 \mathrm{ccm}$ Blut $+400 \mathrm{mg}$ Adeninsulfat bei $40^{\circ}$ in Wasser gelöst mit soviel kaustischer Soda versetzt, um die Lösung zu neutralisieren + hinreichend Ringersche Lösung, um. eine Menge von 1 Liter zu ergeben. Diese Lösung wurde für die Perfusion gebraucht.

3. Eine wie Nr. 2 hergestellte und behandelte Lösung, die aber nicht zur Perfusion diente.

Lösung Nr. 2 wurde durch die isolierten Hinterbeinmuskeln eines Hundes hindurchgeleitet, indem sie durch die Aorta aldominalis hinein und durch die vena cava inferior herausströmte. Die Perfusion wurde in der durch Burian beschriebenen Art gemacht und dauerte eine Stunde. Während des größten Teils. dieser Zeit bewiesen Zusammenziehungen, daß der Muskel lebte. $900 \mathrm{ccm}$ der Flüssigkeit wurden wiedererhalten.

Die Perfusionsflüssigkeit samt dem Spülwasser, ebenso wie die Kontrollflüssigkeit Nr. 3 wurden dann einer chemischen Prüfung unterzogen. Nach Entfernung der Eiweißkörper durch Hitzekoagulation wurden die Purinkörper zuerst als Kupfer-, dann als Silberverbindungen niedergeschlagen. Das Filtrat von Chlorsilber wurde verdampft, um den Überschuß von Salzsäure zu entfernen. Der so von der Perfusionsflüssigkeit erhaltene Rückstand löste sich glatt in Wasser bei $40^{\circ}$. Es blieb nur eine minimale Spur ungelöster Substanz, welche die Andeutung einer Murexidreaktion gab, aber es war so wenig, daß eine befriedigende Untersuchung nicht gemacht werden konnte. Die Lösung, die den größten Teil der Purinsubstanzen enthielt (Hypoxanthinfraktion), wurde mit einer sehr kleinen Menge Tierkohle behandelt, um eine Spur von suspendierter Substanz wegzubringen, und das Adenin mit Pikrinsäure ausgefällt.

\begin{tabular}{|c|c|c|c|c|}
\hline \multirow{2}{*}{$\begin{array}{l}\text { Ursprüng- } \\
\text { liches } \\
\text { Adenin }\end{array}$} & \multicolumn{2}{|c|}{ Adeninpikrat } & \multicolumn{2}{|c|}{$\begin{array}{c}\text { Daraus berechnet } \\
\text { Adenin }\end{array}$} \\
\hline & $\begin{array}{l}\text { aus der durch- } \\
\text { geleiteten } \\
\text { Flüssigkeit }\end{array}$ & $\begin{array}{c}\text { aus der } \\
\text { Kontrollprobe }\end{array}$ & $\begin{array}{c}\text { aus der durch- } \\
\text { geleiteten } \\
\text { Flüssigkeit }\end{array}$ & $\begin{array}{c}\text { aus der } \\
\text { Kontrollprobe }\end{array}$ \\
\hline $267 \mathrm{mg}$ & $610 \mathrm{mg}$ & $665 \mathrm{mg}$ & $226 \mathrm{mg}$ & $246 \mathrm{mg}$ \\
\hline
\end{tabular}

Die beiden Filtrate vom Adeninpikrat wurden in derselben 
Weise mit denselben Resultaten behandelt wie folgt: Nach Entfernung der Pikrinsäure mit Äther und Schwefelsäure wurde Silberlösung zugesetzt. Dies bewirkte eine Trübung, welche beim Stehen über Nacht einen leichten Niederschlag ergab. Dieser wurde durch Salzsäure auf gewöhnliche Art zersetzt und schließlich eine *Hypoxanthinfraktion* erhalten, wie oben beschrieben. Pikrinsäure erzeugte mit dieser Flüssigkeit sofort einen kleinen Niederschlag, welcher aus heißem Wasser in langen, makroskopischen, seidnen Nadeln krystallisierte, Schmelzpunkt $279^{\circ}$. Die Substanz war unzweifelhaft Adeninpikrat, welches dem ersten Niederschlag mit Pikrinsäure entgangen war. Das letzte Filtrat gab einen Niederschlag mit Silbernitrat und Ammoniak, aber so wenig, daß es aussichtslos schien, es zu identifizieren. Die Menge des Hypoxanthins ist also sicher nicht größer in der Perfusionsflüssigkeit, welche Adenin enthielt, als durch Burian in einer adeninfreien Perfusionsflüssigkeit gefunden wurde.

Bei näherer Überlegung der hier auseinandergesetzten Tatsachen dürfte es scheinen, als ob die Desamidasen und besonders Adenase bei der Bildung der endogenen Harnsäure nicht so sehr beteiligt sind, als man vorausgesetzt hat. Für diese Schlußfolgerung gibt es mehrere voneinander unabhängige Beweise.

1. Die Rattenorganextrakte zeigen nicht die Purinfermente, die zur Bildung von Harnsäure nötig sind, aber Rattenharn enthält Harnsäure.

2. Die Affenorganextrakte zeigen die Purinfermente, die zur Bildung der Harnsäure nötig sind, aber der Affenharn enthält keine oder wenig Harnsäure. ${ }^{1}$ )

3. Ascoli hat eine physiologische Synthese von Harnsäure ausgeführt, welche weder die Gegenwart eines Purinringes noch eines Purinferments voraussetzt. ${ }^{2}$ )

Wir haben nicht den Wunsch, die Wichtigkeit der Purinfermente zu verkleinern, aber wir haben auch keine Neigung, ihnen eine größere Bedeutung beizulegen, als sie verdienen.

1) Wells, loc. cit.

2) Ascoli und Izar, Diese Zeitschrift, Bd. LXII, S. 347. 